\title{
FitorRemediaÇÃo de SOlos ConTAMinados COM HERBICIDAS ${ }^{1}$
}

\author{
Phytoremediation of Herbicide-Polluted Soils
}

\begin{abstract}
PIRES, F.R. ${ }^{2}$, SOUZA, C.M. ${ }^{3}$, SILVA, A.A. ${ }^{3}$, PROCÓPIO, S.O. ${ }^{2}$ e FERREIRA, L.R. ${ }^{3}$
RESUMO - A fitorremediação é uma técnica que objetiva a descontaminação de solo e água, utilizando-se como agente de descontaminação plantas. É uma alternativa aos métodos convencionais de bombeamento e tratamento da água, ou remoção física da camada contaminada de solo, sendo vantajosa principalmente por apresentar potencial para tratamento in situ e ser economicamente viável. Além disso, após extrair o contaminante do solo, a planta armazena-o para tratamento subseqüente, quando necessário, ou mesmo metaboliza-o, podendo, em alguns casos, transformá-lo em produtos menos tóxicos ou mesmo inócuos. A fitorremediação pode ser empregada em solos contaminados por substâncias inorgânicas e/ou orgânicas. Resultados promissores de fitorremediação já foram obtidos para metais pesados, hidrocarbonetos de petróleo, agrotóxicos, explosivos, solventes clorados e subprodutos tóxicos da indústria. A fitorremediação de herbicidas apresenta bons resultados para atrazine, tendo a espécie Kochia scoparia revelado potencial rizosférico para fitoestimular a degradação dessa molécula. Embora ainda incipiente no Brasil, já existem estudos sobre algumas espécies agrícolas cultivadas e espécies silvestres ou nativas da própria área contaminada, com o objetivo de selecionar espécies eficientes na fitorremediação do solo.
\end{abstract}

Palavras-chave: fitorremediação, seleção de plantas, recuperação do solo.

ABSTRACT - Phytoremediation is a technique that uses plants as agents of soil and water decontamination. It is an advantageous alternative to the conventional methods of water pumping and treatment and/or physical removal of the contaminated soil layer since it allows in situ treatment at feasible costs. Also, after extracting the contaminant from the soil, the plant stores it for a subsequent treatment, if necessary, or may metabolically transform it into products that are less or non-toxic. Phytoremediation can be employed in soils contaminated by inorganic or organic substances. Promising phytoremediation results have been obtained in soils contaminated by heavy metals, petroleum hydrocarbons, pesticides, explosives, chlorinated solvents and toxic industrial by-products. Herbicide phytoremediation presents interesting results for atrazine, with Kochia scoparia species revealing rhizospheric potential for phytostimulating molecule degradation. Although this technique is not well known in Brazil, studies have been performed using agricultural crops and wild or native plant species from contaminated areas to select efficient species for soil phytoremediation.

Key words: phytoremediation, plant selection, soil restoration.

\section{INTRODUÇÃO}

Na busca de alternativas para despoluir áreas contaminadas por diversos compostos orgânicos, tem-se optado por soluções que englobam: eficiência na descontaminação, simplicidade na execução, tempo demandado pelo processo e menor custo. Nesse contexto, cresce o interesse pela utilização da biorremediação, caracterizada como uma técnica que

Recebido para publicação em 4.10.2002 e na forma revisada em 11.8.2003.

2 Eng.-Agr., M.S., Doutorando no Curso de Pós-Graduação em Fitotecnia da Universidade Federal de Viçosa - UFV, Bolsista CNPq, 36571-000 Viçosa-MG, <frpires@alunos.ufv.br> e <procopio@alulnos.ufv.br>; ${ }^{3}$ Eng.-Agr., Dr., Prof. Adjunto do Departamento de Fitotecnia da UFV, <cmsouza@mail.ufv.br>, <aasilva@mail.ufv.br> e <1roberto@ufv.br>. 
objetiva descontaminar solo e água por meio da utilização de organismos vivos, como microrganismos e plantas.

Dentro da biorremediação insere-se a fitorremediação, que, segundo Accioly \& Siqueira (2000), envolve o emprego de plantas, sua microbiota associada e de amenizantes (corretivos, fertilizantes, matéria orgânica etc.) do solo, além de práticas agronômicas que, se aplicadas em conjunto, removem, imobilizam ou tornam os contaminantes inofensivos ao ecossistema. A utilização da fitorremediação tem sido estudada e difundida principalmente nos Estados Unidos e na Europa. De acordo com Accioly \& Siqueira (2000), as projeções anuais são de que nos Estados Unidos os gastos com fitorremediação, até 2005, serão de 100-200 milhões de dólares.

Quando comparada com técnicas tradicionais como bombeamento e tratamento, ou remoção fisica da camada contaminada, a fitorremediação tem sido considerada vantajosa, principalmente por sua eficiência na descontaminação e pelo baixo custo (Perkovich et al., 1996; Cunningham et al., 1996).

\section{APLICAÇÃO E MECANISMOS DE FITORREMEDIAÇÃO}

A fitorremediação pode ser usada em solos contaminados com substâncias orgânicas ou inorgânicas, como metais pesados, elementos contaminantes, hidrocarbonetos de petróleo, agrotóxicos, explosivos, solventes clorados e subprodutos tóxicos da indústria (Cunningham et al., 1996). A pesquisa com metais pesados tem sido a mais difundida, como é o caso de algumas espécies que já têm sua eficiência comprovada (Accioly \& Siqueira, 2000). Recentes pesquisas têm difundido a utilização de plantas na fitorremediação de agrotóxicos (Anderson \& Coats, 1995; Cunningham et al., 1996; Fernandez et al., 1999; Vose et al., 2000; Pires et al., 2001).

Em geral, é mais dificil trabalhar com contaminantes orgânicos, em razão da diversidade molecular, da complexidade de análise e das constantes transformações a que estão sujeitos. Os metais pesados, por exemplo, são mais facilmente quantificados e raramente formam metabólitos intermediários no solo, como ocorre na biodegradação dos contaminantes orgânicos (Cunningham et al., 1996). Assim, as pesquisas com compostos orgânicos contaminantes de solo exigem técnicas especializadas e de custo elevado, envolvendo o uso de elementos marcados e sofisticada instrumentação analítica.

A utilização da fitorremediação é baseada na seletividade, natural ou desenvolvida, que algumas espécies exibem a determinados tipos de compostos ou mecanismos de ação. Esse fato é de ocorrência comum em espécies agrícolas e daninhas, tolerantes a certos herbicidas. A seletividade deve-se ao fato de que os compostos orgânicos podem ser translocados para outros tecidos da planta e subseqüentemente volatilizados; podem ainda sofrer parcial ou completa degradação ou ser transformados em compostos menos tóxicos, especialmente menos fitotóxicos, combinados e/ou ligados a tecidos das plantas (compartimentalização) (Accioly \& Siqueira, 2000; Scramin et al., 2001). A maioria dos orgânicos parece sofrer algum grau de transformação nas células das plantas antes de serem isolados em vacúolos ou ligarem-se a estruturas celulares insolúveis, como a lignina (Salt et al., 1998). A capacidade de metabolização do agrotóxico a um composto não-tóxico (ou menos tóxico) à planta e ao ambiente é o princípio da fitodegradação. Outra possibilidade é a fitoestimulação, na qual há o estímulo à atividade microbiana, promovido pela liberação de exsudatos radiculares, que atua degradando o composto no solo, o que caracteriza, em algumas plantas, a aptidão rizosférica para a biorremediação de compostos tóxicos.

A absorção de compostos orgânicos como herbicidas pelas plantas é afetada pelas propriedades químicas do composto, pelas condições ambientais e pelas características da espécie vegetal (Burken \& Schnoor, 1996). Para ser translocado, o produto químico deve passar pelo simplasto da endoderme. Para certas características das plantas e condições ambientais, a absorção radicular de xenobióticos da água está diretamente relacionada ao logaritmo do coeficiente de partição octanol-água $\left(K_{o w}\right)$ do composto; além dessa característica, interferem ainda a constante de acidez $\left(p K_{a}\right)$ e a sua concentração (Alkorta $\&$ Garbisu, 2001). De acordo com Brigss et al. (1982), o fluxo 
transpiratório, fundamental para promover o carreamento do químico absorvido para a parte aérea das plantas, levando à fitodegradação, é maior quando o Log $K_{o w}$ do pesticida varia de 0,5 a 3,0, sendo maior a absorção quando o valor de $\log K_{\text {ow }}$ é igual a 2,1. Compostos que são mais hidrofóbicos, com $\log K_{o w}>2,1$, ligam-se às membranas lipídicas das raízes antes de entrarem no xilema. Compostos que são menos hidrofóbicos, com valores de Log $K_{o w}<2,1$, não passam através das membranas lipídicas associadas com as camadas da epiderme das raízes. Essa ligação ou exclusão leva a um menor fluxo transpiratório sob valores de $\log K_{o w}$ que se distanciam de 2,1.

\section{EXEMPLOS DE FITORREMEDIAÇÃO DE HERBICIDAS}

O desenvolvimento de moléculas herbicidas com efeito residual longo possibilitou o controle efetivo de plantas daninhas por um período de tempo maior, reduzindo com isso o número de aplicações. No entanto, tem-se observado a ocorrência de fitotoxicidade em culturas sensiveis (carryover) plantadas após a utilização desses herbicidas, cujo efeito residual varia de alguns meses até três anos ou mais. Esse fenômeno tem sido observado para os herbicidas atrazine e imazaquin (Siqueira et al., 1991), além de outros, como tebuthiuron, fomesafen e trifloxysulfuronmetyl. Nessa situação, torna-se possível e recomendável o emprego de espécies vegetais na descontaminação dos solos.

Existe ainda o problema ambiental ocasionado pela lixiviação das moléculas de herbicidas ou de seus metabólitos para camadas mais profundas no perfil do solo, podendo atingir o lençol de água subterrâneo. Quando o produto permanece por mais tempo no solo sem ser adsorvido aos colóides do solo, degradado e/ou mineralizado, a possibilidade de lixiviação é maior. Isso comprova que o comportamento do herbicida no solo será influenciado, entre outros fatores, pelas suas próprias características.

Solos contaminados com herbicidas apresentam certas limitações à fitorremediação, quando comparados com aqueles contaminados com outros contaminantes orgânicos ou inorgânicos, por serem, muitas vezes, tóxicos às plantas, principalmente onde ocorrem misturas desses compostos (aumento do espectro de ação). Todavia, alguns trabalhos relatam a contribuição de plantas, estimulando o efeito rizosférico e acelerando a mineralização de alguns herbicidas. Anderson et al. (1994) demonstraram que a degradação de atrazine, metolachlor e trifluralin foi significativamente maior em solos rizosféricos de $K$. scoparia que em solos não-vegetados. Resultados semelhantes foram obtidos por Perkovich et al. (1996), os quais observaram que a mineralização de ${ }^{14} \mathrm{C}$-atrazine em uma mistura de atrazine e metolachlor foi significativamente maior em solos rizosféricos de $K$. scoparia que em solos sem a presença de plantas.

Em experimento realizado com o objetivo de avaliar o efeito de solos rizosféricos de 15 espécies de plantas sobre a mineralização de ${ }^{14} \mathrm{C}$-atrazine e ${ }^{14} \mathrm{C}$-metolachlor, Anderson \& Coats (1995) constataram que $K$. scoparia apresentou a maior mineralização de ${ }^{14} \mathrm{C}$-atrazine (62,1\% do aplicado) dentre as espécies testadas, seguida por Carduus nutans $(33,1 \%)$ e Nepeta cataria $(24,1 \%)$. Para a mineralização do metolachlor nenhuma das amostras de solo rizosférico exibiu resposta positiva.

Em estudo para seleção de espécies capazes de descontaminar solos de quatro locais contaminados com agroquímicos em Iowa, EUA, Kruger et al. (1997) observaram que ocorreu rápida mineralização em três dos oito solos testados, com maior quantidade ocorrendo em solo rizosférico (35\% da atrazine aplicada após nove semanas). As plantas causaram um impacto positivo na dissipação de atrazine no solo, sendo significativamente menor a quantidade de atrazine extraível de solos vegetados com Kochia do que de solos sem vegetação.

Estudos realizados por Burken \& Schnoor (1996) indicaram que Populus deltoides nigra DN34 foi capaz de absorver a maior parte da atrazine aplicada que não foi totalmente sorvida à fração orgânica do solo, sem efeitos adversos detectáveis às árvores da espécie testada.

Wilson et al. (2000a) testaram duas espécies ornamentais (Acorus gramenius e Pontederia cordata) em relação à fitotoxicidade, absorção e distribuição de ${ }^{14} \mathrm{C}$-simazine e verificaram que a atividade de simazine na solução foi reduzida a 45 e $34 \%$, em sete dias na 
presença de $A$. grameniuse $P$. cordata, respectivamente, sugerindo serem elas eficientes na fitorremediação de simazine. De modo semelhante, Wilson et al. (2000b) constataram que a espécie de cacto Typha latifolia foi capaz de reduzir 34 e $64 \%$ da atividade do fungicida metalaxyl e do herbicida simazine, respectivamente, cultivado em solução após sete dias, apesar da redução observada na produção de matéria fresca, proporcionada pela simazine. Os resultados indicaram a possibilidade de incorporar essa espécie em esquema de fitorremediação de metalaxyl e simazine.

Rice et al. (1997) encontraram respostas que sustentam a hipótese de que a presença de vegetação aquática tolerante a herbicidas pode acelerar a remoção e biotransformação de metolachlor e atrazine de águas contaminadas com esses herbicidas. Após 16 dias de incubação, apenas 1,44, 4,6 e 22,7\% do ${ }^{14} \mathrm{C}$-metolachlor aplicado permaneceram na água da superfície do sistema contendo Ceratophyllum demersun, Elodea canadensis e Lemna minor, respectivamente, enquanto 61\% do ${ }^{14} \mathrm{C}$-metolachlor aplicado persistiu na superfície da água dos sistemas sem plantas. Para ${ }^{14} \mathrm{C}$-atrazine, 41,3 e 63,2\% do aplicado permaneceu nos sistemas vegetados contendo C. demersum e E. canadensis, respectivamente, e $85 \%$ foram detectados na água com $L$. minor.

Em outro trabalho, desenvolvido por Fernandez et al. (1999), observou-se que as espécies Canna xgeneralis e Pontaderia cordata sofreram reduções mínimas no crescimento e nos atributos fotossintéticos avaliados (fotossintese, respiração e fotorrespiração), quando cultivadas em sistemas onde o herbicida orizalin estava presente, indicando que, nessas condições, essas plantas poderiam ser mais bem estudadas quanto ao potencial fitorremediador.

Como se observa, a maioria das informações geradas até o momento é proveniente de pesquisas realizadas nos Estados Unidos e na Europa. No Brasil, praticamente inexistem trabalhos que envolvam a fitorremediação de solos contaminados por herbicidas. A pesquisa nessa área encontra-se ainda incipiente. Um dos raros trabalhos relatados é o de Scramin et al. (2001), no qual se buscou identificar espécies vegetais tolerantes a herbicidas em áreas de cultivo de cana-de-açúcar no Estado de São Paulo, para utilização em programas de fitorremediação. Observaram a ocorrência mais freqüente de oito espécies de plantas daninhas persistentes, supostamente tolerantes aos herbicidas mais comumente utilizados nas áreas de cultivo de cana-de-açúcar na região, sendo elas: Cynodon dactylon, Cyperus rotundus, Digitaria horizontalis, Commelina benghalensis, Brachiaria decumbens, Euphorbia heterophyla, Chamaesyce hirta e Chamaesyce hyssopifolia.

Apesar da dificuldade de identificar espécies que reúnem, além da capacidade fitorremediadora, características desejáveis do ponto de vista agronômico, como fácil propagação e também fácil controle ou erradicação da área, um dado importante e animador, evidenciado por Arthur et al. (2000), que comprova a possibilidade de utilização da fitorremediação, é que, nos solos rizosféricos de $K$. scoparia, a meiavida da atrazine foi de 50 dias e, nos solos nãovegetados, de 193 dias. Esses resultados demonstram claramente o potencial da utilização de plantas terrestres e aquáticas como fitorremediadoras de agrotóxicos.

\section{PRÉ-REQUISITOS PARA UTILIZAÇÃO DA FITORREMEDIAÇÃO}

Antes da implantação de programas de fitorremediação, as características físicoquímicas do solo e do contaminante devem ser conhecidas, bem como sua distribuição na área. Qualquer fator que venha a interferir negativamente no desempenho das plantas fitorremediadoras deve ser controlado ou minimizado, para favorecer sua atuação descontaminante.

É desejável que as plantas que apresentem potencial para fitorremediação possuam algumas características que devam ser usadas como indicativos para seleção. Com base nas análises apresentadas por diversos autores (Ferro et al., 1994; Perkovich et al., 1996; Cunningham et al., 1996; Newman et al., 1998; Accioly \& Siqueira, 2000; Vose et al., 2000), essas características devem ser:

- capacidade de absorção, concentração e/ou metabolização e tolerância ao contaminante;

- retenção do contaminante nas raízes, no caso da fitoestabilização, como oposto à 
transferência para a parte aérea, evitando sua manipulação e disposição;

- sistema radicular profundo e denso;

- alta taxa de crescimento e produção de biomassa;

- capacidade transpiratória elevada, especialmente em árvores e plantas perenes;

- fácil colheita, quando necessária a remoção da planta da área contaminada;

- elevada taxa de exsudação radicular;

- resistência a pragas e doenças;

- fácil aquisição ou multiplicação de propágulos;

- fácil controle ou erradicação;

- capacidade de desenvolver-se bem em ambientes diversos; e

- ocorrência natural em áreas poluídas (importante na identificação, porém não é prérequisito).

Naturalmente, torna-se dificil reunir todas essas características numa só planta, porém aquela que for selecionada deve reunir o maior número delas. Outro aspecto a ser observado é que, embora a maioria dos testes avalie plantas isoladas, várias espécies podem ser usadas em um mesmo local, ou ao mesmo tempo ou subseqüentemente, para remover mais de um contaminante (Miller, 1996).

\section{POTENCIALIDADES E LIMITAÇÕES DA FITORREMEDIAÇÃO}

\section{Potencialidades}

A fitorremediação apresenta elevado potencial de utilização, devido às vantagens que apresenta em relação às outras técnicas de remediação de contaminantes do solo. Com base nos relatos de Cole et al. (1995), Cunningham et al. (1996) e Vose et al. (2000), essas vantagens são:

- menor custo em relação às técnicas tradicionalmente utilizadas envolvendo a remoção do solo para tratamento ex situ. Esta, segundo Cunningham et al. (1996), é uma das principais vantagens da fitorremediação;
- na maioria dos casos, os equipamentos e suprimentos empregados no programa de fitorremediação são os mesmos utilizados na agricultura. Logo, quando a fitorremediação é implantada em áreas agrícolas, o custo deve ser ainda menor;

- os compostos orgânicos podem ser degradados a $\mathrm{CO}_{2}$ e $\mathrm{H}_{2} \mathrm{O}$, removendo toda a fonte de contaminação, não havendo, nessa situação, a necessidade de retirada das plantas fitorremediadoras da área contaminada. Esta situação não é válida para metais pesados;

- plantas são mais fáceis de ser monitoradas do que microrganismos, por exemplo;

- as propriedades biológicas e físicas do solo são mantidas e, não raro, até melhoradas;

- incorporação de matéria orgânica ao solo, quando não há necessidade de retirada das plantas fitorremediadoras da área contaminada;

- fixação de nitrogênio atmosférico, no caso de leguminosas;

- plantas ajudam no controle do processo erosivo, eólico e hídrico. Nesse último caso, evitam o carreamento de contaminantes com a água e com o solo e, por conseguinte, reduzem a possibilidade de contaminação de lagos e rios;

- pode-se considerar, também, que a planta reduz o movimento descendente de água contaminada de camadas superficiais do solo para o lençol freático;

- plantas são mais favoráveis, esteticamente, do que qualquer outra técnica de biorremediação e podem ser implementadas com mínimo distúrbio ambiental, evitando escavações e tráfego pesado;

- utiliza energia solar para realizar os processos; e

- tem alta probabilidade de aceitação pública.

Considerando esses fatores e o mais baixo custo esperado para a fitorremediação, ela pode ser usada em maior escala do que seria possivel no caso de outros métodos.

\section{Limitações}

A técnica da fitorremediação não é aplicável universalmente nem é um sistema perfeito. Ela 
oferece muitos aspectos positivos, mas também existem inconvenientes. As limitações da fitorremediação de compostos orgânicos em geral e de agrotóxicos, relatadas por Narayanan et al. (1996), Cunningham et al. (1996), Miller (1996) e Macek et al. (2000), são:

- dificuldade na seleção de plantas para fitorremediação, principalmente em relação à descontaminação de herbicidas de amplo espectro de ação ou em misturas no solo;

- o tempo requerido para obtenção de uma despoluição satisfatória pode ser longo (usualmente mais de uma estação de crescimento);

- o contaminante deve estar dentro da zona de alcance do sistema radicular;

- clima e condições edáficas podem restringir o crescimento de plantas fitorremediadoras;

- elevados níveis do contaminante no solo podem impedir a introdução de plantas no sítio contaminado;

- na fitorremediação de orgânicos, as plantas podem metabolizar os compostos, o que não quer dizer que eles serão completamente mineralizados. Em alguns casos, os metabólitos podem ser mais problemáticos do que os compostos originais;

- potencial de contaminação da cadeia alimentar;

- necessidade de disposição da biomassa vegetal, quando ocorre a fitoextração de poluentes não-metabolizáveis ou metabolizados a compostos também tóxicos;

- possibilidade de a planta fitorremediadora tornar-se planta daninha; e

- melhoria nas condições do solo pode ser requerida, incluindo a quelação do contaminante para facilitar sua absorção pelas plantas, devido à quebra de pontes de ligação com partículas do solo.

Apesar de existirem limitações, os benefícios apresentados pela fitorremediação a tornam uma técnica promissora. Contudo, ela requer ação conjunta de profissionais de diversas áreas, no intuito de identificar espécies capazes de atuar na descontaminação de solos, principalmente em condições edafoclimáticas brasileiras.

\section{LITERATURA CITADA}

ACCIOLY, A. M. A.; SIQUEIRA, J. O. Contaminação química e biorremediação do solo. In: NOVAIS, R. F.; ALVAREZ V.; V. H.; SCHAEFER, C. E. G. R. Tópicos em ciência do solo. Viçosa: Sociedade Brasileira de Ciência do Solo, 2000. v. 1. p. 299-352.

ANDERSON, T. A.; COATS, J. R. Screening rhizosphere soil samples for the ability to mineralize elevated concentrations of atrazine and metolachlor. J. Environ. Sci. Health, B, v. 30, p. 473-484, 1995.

ANDERSON, T. A.; KRUGER, E. L.; COATS, J. R. Enhanced degradation of a mixture of three herbicides in the rhizosphere of a herbicide-tolerant plant. Chemosphere, v.28, p.1551-1557, 1994.

ALKORTA, I.; GARBISU, C. Phytoremediation of organic contaminants in soils. Biores. Technol., v. 79, p. 273-276, 2001 .

ARTHUR, E. L. et al. Degradation of an atrazine and metolachlor herbicide mixture in pesticide-contaminated soils from two agrochemical dealerships in Iowa. Water, Air, Soil Poll., v. 119, p. 75-90, 2000.

BRIGSS, G. G.; BROMILOW, R. H; EVANS, A. A. Relationship between lipphilicity and root uptake and translocation of non-ionized chemicals by barley. Pestc. Sci., v. 13, p. 495-504, 1982.

BURKEN, J. G.; SCHNOOR, J. L. Phytoremediation: plant uptake of atrazine and role of root exudates. J. Environ. Engin., v. 122, p. 958-963, 1996.

COLE, M. A.; ZHANG, L.; LIU, X. Remediation of pesticide-contaminated soil by planting and compost addition. Compost. Sci. Utiliz., v. 3, p. 20-30, 1995.

CUNNINGHAM, S. D.; ANDERSON, T. A.; SCHWAB, A. P. Phytoremediation of soils contaminated with organic pollutants. Adv. Agron., v. 56, p. 55-114, 1996.

FERNANDEZ, R. T. et al. Evaluating semiaquatic herbaceous perennials for use in herbicide phytoremediation. J. Am. Soc. Hortic. Sci., v. 124, p. 539544, 1999.

FERRO, A. M.; SIMS, R. C.; BUGBEE, B. Hycrest crested wheatgrass accelerates the degradation of pentachlorophenol in soil. J. Environ. Qual., v. 23, p. 272-279, 1994.

KRUGER, E. L. et al. Atrazine degradation in pesticidecontaminated soils: phytoremediation potential. In: PHYTOREMEDIATION OF SOIL AND WATER CONTAMINANTS, 1997, Washington, DC: ACS Symposium Series... Washington, DC: American Chemical Society, 1997. p. 54-64. 
MACEK, T.; MACKOVÁ, M.; KÁŠ, J. Exploitation of plants for the removal of organics in environmental remediation. Biotechnol. Adv., v. 18, p. 23-34, 2000.

MILlER, R. R. Phytoremediation. 1996. Disponível em http://www.gwrtac.org. Disponível em 18 jun. 2001.

NARAYANAN, M. et al. Experimental and modeling studies of the fate of trichlorethylene in a chamber with alfafa plants. 1996. Online. Disponível em http:// www.engg.ksu.edu/HSRC/home.html. Disponível em 18 jun. 2001.

NEWMAN, L. A. et al. Phytoremediation of organic contaminants: a review of phytoremediation research at the university of Washington. J. Soil Contamin., v. 7, p. 531$542,1998$.

PERKOVICH, B. S. et al. Enhanced mineralization of $\left[{ }^{14} \mathrm{C}\right]$ atrazine in $K$. scoparia rhizosferic soil from a pesticidecontaminated site. Pestic. Sci., v. 46, p. 391-396, 1996.

PIRES, F. R. et al. Uso da fitorremediação na descontaminação do solo. In: ENCONTRO REGIONAL DE BOTÂNICOS, 23., 2001, Viçosa, MG. Resumos... Viçosa: Universidade Federal de Viçosa, 2001. p. 104.

RICE, P. J.; ANDERSON, T. A.; COATS, J. R. Phytoremediation of herbicide-contaminated surface water with aquatic plants. In: PHYTOREMEDIATION OF SOIL AND WATER CONTAMINANTS, 1997, Washington, DC. ACS Symposium Series... Washington, DC: American Chemical Society, 1997. p.133-151.
SALT, D. E.; SMITH, R. D.; RASKIN, I.

Phytoremediation. Ann. Rev. Plant Physiol. Plant Molec. Biol., v. 49, p. 643-668, 1998.

SCRAMIN, S.; SKORUPA, L. A.; MELO, I. S. Utilização de plantas na remediação de solos contaminados por herbicidas - levantamento da flora existente em áreas de cultivo de cana-de-açúcar. In: MELO, I. S. et al.

Biodegradação. Jaguariúna, SP: EMBRAPA Meio Ambiente, 2001. p. 369-371.

SIQUEIRA, J. O.; SAFIR, G. R.; NAIR, M. G. VAmycorrhizae and mycorrhizal stimulating isoflavonoid compounds reduce plant herbicidae injury. Plant Soil, v. 34, p. 233-242, 1991.

VOSE, J. M. et al. Leaf water relations and sapflow in Eastern cottonwood (Populus detoides Bartr.) trees planted for phytoremediation of a groundwater pollutant. Intern. J. Phytoremediaton., v. 2, p. 53-73, 2000.

WILSON, P. C.; WHITWELL, T.; KLAINE, S. J. Phytotoxicity, uptake, and distribution of ${ }^{14} \mathrm{C}$-simazine in Acorus gramenius and Pontederia cordata. Weed Sci., v. 48 , p. 701-709, 2000a.

WILSON, P. C.; WHITWELL, T.; KLAINE, S. J. Metalaxyl toxicity, uptake, and distribution in several ornamental plant species. Arch. Environ. Contam. Toxicol., v. 39, p. 282288, 2000b. 